\title{
Hematologic and systemic metabolic alterations due to Mediterranean type II G6PD deficiency in mice
}

Angelo D’Alessandro ${ }^{1}$, Heather L Howie ${ }^{2,3}$, Ariel M. Hay ${ }^{2,3}$, Karolina H. Dziewulska, Benjamin Brown $^{1}$, Matthew J Wither ${ }^{1}$, Matthew Karafin ${ }^{4}$, Elizabeth F. Stone ${ }^{5}$, Steven L Spitalnik ${ }^{5}$, Eldad A Hod $^{5}$, Richard O Francis ${ }^{5}$, Xiaoyun Fu ${ }^{3}$, Tiffany Thomas ${ }^{5}$, James C Zimring ${ }^{2,3 *}$

${ }^{1}$ University of Colorado Denver-Anschutz Medical Campus, Aurora, CO

${ }^{2}$ Univeristy of Virginia Department of Pathology and Carter Immunology Center, Charlottesville, VA

${ }^{3}$ Bloodworks NW Research Institute, Seattle, WA

${ }^{4}$ Blood Center of Wisconsin, Milwaukee WI

${ }^{5}$ Columbia University, Department of Pathology and Cell Biology, New York, NY

*To whom all correspondence should be addressed

Please address correspondence to: James C. Zimring, University of Virginia School of Medicine, P.O. Box 801386, Charlottesville, VA 22908 email: jcz2k@virginia.edu

Running Title: Metabolic Alterations due to G6PD Deficiency

Scientific Category: Red Cells, Iron, Erythropoiesis 


\begin{abstract}
:
Deficiency of Glucose 6 phosphate dehydrogenase (G6PD) is the single most common enzymopathy, present in approximately 400 million humans (e.g. 5\% of humans). Its prevalence is hypothesized to be due to conferring resistance to malaria. However, G6PD deficiency also results in hemolytic sequelae from oxidant stress. Moreover, G6PD deficiency is associated with kidney disease, diabetes, pulmonary hypertension, immunological defects, and neurodegenerative diseases. To date, the only available mouse models have decreased levels of G6PD due to promoter mutations, but with stable G6PD. However, human G6PD mutations are missense mutations that result in decreased enzymatic stability. As such, this results in very low activity in red blood cells and platelets that cannot synthesize new protein. To generate a more accurate model, the human sequence for a severe form of G6PD deficiency (Med -) was knocked into the murine G6PD locus. As predicted, G6PD levels were extremely low in RBCs and deficient mice have increased hemolytic sequalae to oxidant stress. G6PD levels were mildly decreased in nonerythroid organs, consistent with what has been observed in humans. Juxtaposition of G6PD deficient and wild-type mice revealed altered lipid metabolism in multiple organ systems. Together, these findings both establish a new mouse model of G6PD deficiency that more accurately reflects human G6PD deficiency and also advance our basic understanding of altered metabolism in this setting.
\end{abstract}




\section{Introduction:}

Glucose-6-phosphate dehydrogenase (G6PD) is the first and rate determining enzyme in the pentose phosphate pathway (PPP), which utilizes glucose to generate NADPH; the latter is the major reducing equivalent that fuels multiple pathways by which cells handle oxidative stress. Deficiency in the activity of G6PD is the single most common enzymopathy in humans, estimated to be present in approximately 400 million people worldwide(1). The complete absence of G6PD is not compatible with life, and the vast majority of mutations leading to G6PD deficiency in humans are missense mutations leading to an unstable G6PD enzyme. Based upon the mutation and resulting G6PD levels, disease can range from mild to severe deficiency $(2,3)$. RBCs from humans with G6PD deficiency are particularly susceptible to oxidant stress for two prevailing reasons. First, because RBCs lack mitochondria, the PPP is their main source of NADPH. Second, mature RBCs are unable to synthesize new proteins. When G6PD deficient humans encounter an illness or consume a drug or food that increases oxidant stress (e.g., antimalarial quinone drugs or fava beans), they can manifest symptoms of actue hemolysis, ranging from mild to lethal $(4,5)$. Recent findings have also linked G6PD deficiency to many other diseases outside the RBC compartment, including immunological(1), cardiovascular(6), endocrine(7), renal(7), neurological(8), and pulmonary pathologies(9).

Because G6PD deficiency is so prevalent in humans, and because its biology remains poorly understood, a translatable animal model of G6PD deficiency is essential. Two separate mouse models of G6PD deficiency have been described(10,11), both derived by random mutagenesis. Importantly, both result in altered levels of G6PD expression, but with a normal amino acid sequence and normal protein stability $(10,11)$. As such, they do not recapitulate the human situation where young RBCs have high levels of G6PD activity, which then diminish as the RBCs age. To our knowledge, no mouse model has been described with an unstable G6PD enzyme.

Type II G6PD deficiency is the most severe form that lacks ongoing non-spherocytic hemolytic anemia at baseline. The best studied type II deficiency is the "Mediterranean variant" that results from a Ser 188 to Phe mutation $(12,13)$. Herein, we describe a novel mouse in which the genomic sequence for the human "Mediterranean-Type" G6PD-deficient mutant was knocked into the murine G6PD locus, thus recapitulating the human enzymopathy in a murine system. The 
phenotype of this mouse resembles that of humans with G6PD deficiency, in that the mice are healthy at baseline but demonstrate hemolysis upon challenge with oxidant stress. A detailed analysis of these mice was performed, under normal and stressed conditions, including metabolomics profiling of multiple organs. In addition to describing a new model, these findings provide novel understanding of the metabolic consequences of G6PD deficiency in multiple organ systems, as well as unique insights into the biochemical mechanisms by which oxidant stress alters RBCs, both in the G6PD-normal and G6PD-deficient states. 


\section{Results:}

\section{Generation of a Novel Murine Model of Human G6PD Deficiency}

A targeting construct was generated to insert the human type II Mediterranean variant [hMed(-)] variant directly into the murine G6PD locus (Figure 1A.1). To maintain genetic structure, the entire genomic sequence of human $\mathrm{hMed}(-)$ was utilized from exons 3-12. Out of concern for disrupting genomic regulatory elements in the proximal murine sequence, exons 1 and 2, and introns 1 and 2 of the murine sequence were left unaltered. As such, the final G6PD gene product is the hMed(-) form (Ser188Phe) that also has two amino acids in the N-terminus from the murine sequence (Figure 1B).

Out of concern that the modification may be embryonic lethal, and also to allow additional experimental flexibility, the genetic construct was designed for conditional expression of the hMed(-) gene. Wild-type mouse G6PD cDNA (exons 3-13) were inserted upstream of the human gene sequence and were flanked with LoxP sites. This construct is designed to express wild-type murine G6PD until it is exposed to CRE recombinase, at which time the mouse cDNA is excised and the human genomic sequence for the hMed(-) G6PD is expressed. (Figure 1A.3-1A.4). This mouse is called "Con-hMed(-)", and was generated using ES cells from a C57BL/6 background. Southern blot analysis was carried out both on modificed ES cells and also on founder mice to confirm the genetic alteration was made (Supplementary Figure 1A).

The Con-hMed(-) mouse was bred with mice that expressed germline CRE, and then were crossed with wild-type B6 to remove the CRE gene (Figure 1A.4), resulting in a germline hMed() mouse (called G6PDMed- ) . Correct excision of the floxed region was confirmed by PCR (data

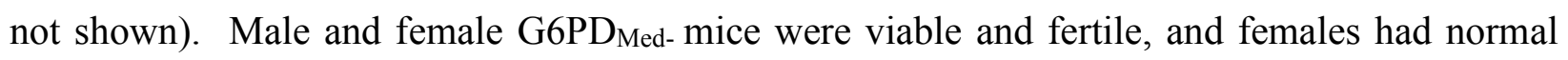
fecundity. As in humans, the murine G6PD locus is on the X-chromosome; thus, heterozygous female $\mathrm{G} \mathrm{PD} \mathrm{D}_{\mathrm{Med}}$ - mice were bred with wild-type males, such that $50 \%$ of the male offspring were hemizygous for hMed(-) and the other $50 \%$ were wild-type. In this way, G6PD Med- mice were compared to littermate control wild-type mice.

RBCs from G6PD Med- mice had only 5\% of the G6PD activity compared to wild-type controls (Figure 1C). The decreased G6PD activity was not due to decreased gene expression, as there was no difference in the amount of hMed(-) G6PD mRNA in G6PDMed- compared to wildtype G6PD mRNA in B6 mice (Figure 1D). However, Western blot analysis of the cytoplasm from RBCs using an antibody that reacts with both human and murine G6PD demonstrated only 


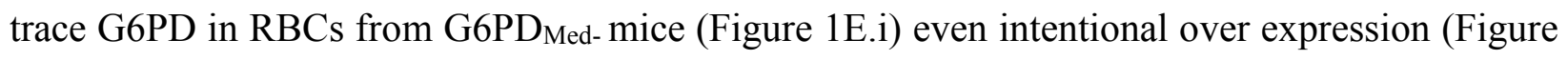
1E.ii.) This was not due to differences in loading the gel, as equivalent levels of beta actin were detected in all samples.

Peripheral blood was analyzed for standard metrics of RBC biology. There was no statistical differences in the values of RBC number, Hemoglobin concentration, hematocrit, mean corposcular volume, red cell distribution width, or reticulocyte counts between G6PD ${ }_{\text {Med- }}$ and wild-type mice (Supplementary figure 2).

\section{-hMed(-) RBCs have decreased PPP activity ex vivo}

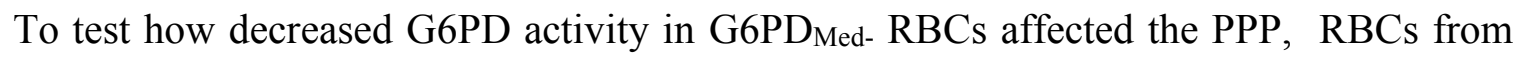

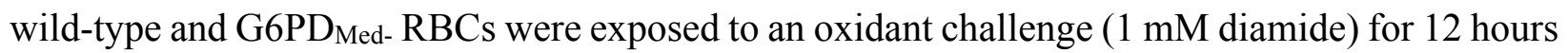
in the presence of $[1,2,3]-{ }^{13} \mathrm{C}_{3}$-glucose (Figure 2A). Multivariate analyses, including partial least square-discriminant analysis (PLS-DA - Figure 2B) and hierarchical clustering analysis (Figure 2C), showed significantly distinct metabolic phenotypes between G6PD Med- RBCs and wild-type RBCs. A version of the heat map in Figure 2C, which also includes the top 50 significant metabolites (and isotopologues) that are significant by repeat measure ANOVA, is provided as Supplementary Figure 3. Results are also provided in tabular form in Supplementary Table 1. G6PDMed- mice had a substantially decreased ratio of ${ }^{13} \mathrm{C}_{2} /{ }^{13} \mathrm{C}_{3}$ lactate isotopologues, indicating decreased flux through the PPP (Figure 2.D). Consistent with this interpretiaion of the decreased

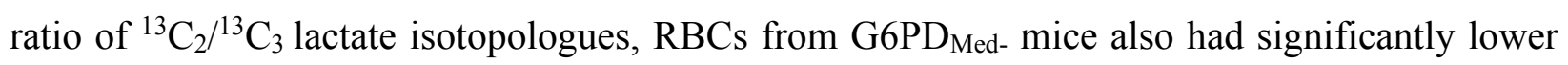
ribose phosphate (Figure 2E), which is an end product of the PPP, as well as ${ }^{13} \mathrm{C}_{3^{-}}$ phosphogluconate, which is an intermdiate of the oxidative phase of the PPP. The untargeted metabolomics analyses also demonstrate that, in addition to alterations in glycolysis and PPP, hMed(-) RBCs had significantly altered redox regulated pathways, including methionine metabolism and purine oxidation (Figure 3). As these are steady state levels, one cannot infer changes in sythensis or consumption, only that levels are different.

\section{RBCs from G6PD $D_{\text {Med- }}$ mice have increased hemolysis in response in vivo to pharmacological oxidant stress}

To test the hypothesis that G6PDMed- mice were more sensitive to oxidant stress in vivo, a method was used by which the complete blood compartment of G6PDMed- mice and wild-type wer 
biotinylated (14). This is the equivalent of an in vivo pulse chase experiment, allowing the determination of RBC lifespan without interference of erythropoiesis, as newly generated RBCs are not biotinylated. Baseline studies determined that the normal circulatory lifespan of G6PDMedRBCs was indistinguishable from that of wild-type controls (Figure 4A).

Phenylhydrazine (PHZ) is a classic chemical to induce oxidant stress in vivo, and is known to cause intravascular hemolysis in human G6PD-deficient subjects(15). PHZ induced a

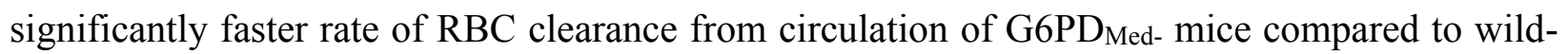
type controls (Figure 4B - Supplementary Table 2). In addition, G6PD Med- but not wild-type controls displayed significant hemoglobinuria (Figure 4C). Metabolomics analyses were performed in blood from the mice at baseline (prior to biotinylation), at day 1 (after biotinylation), at day 2 (middle of PHZ challenge), and at days 5 and 7 (post-PHZ challenge). Results are summarized in the heat map in Figure 4D, with more extensive information provided in tabulated and vectorial forms (including metabolite names) in Supplementary Figure 4.

The ratio of steady state glucose 6-phosphate (G6P) to 6-phospho-gluconate (6PG) (i.e., the substrate and downstream metabolite of G6PD, respectively), is a proxy for G6PD activity(16) (Figure 4E). Consistent with the ex vivo measurements described above, in vivo measurements of 6PG/G6P ratios were significantly lower at baseline and following PHZ exposure in G6PD Medmice, compared to wild-type animals (Figure 4E). Repeated measure ANOVA of time course data further highlighted that the top four metabolites that significantly differed between the two groups as a function of PHZ stimulation were PPP metabolites (i.e., G6P, 6PG, ribose phosphate and isobaric isomers, sedoheptulose phosphate; Figure 4F). Other metabolites related to redox homeostasis were significantly affected by PHZ stimulation, as a function of G6PD status, including glutathione, oxylipins (HETEs), proteolysis markers (e.g., hydroxyproline, methyllysine), and other antioxidant metabolites (e.g., carnosine, ergothioneine; Figure 4E, Supplementary Figure 4). These changes between G6PD $_{\text {Med- }}$ and wild-type mice were most evident after PHZ injection (a heat map of the top 25 significant metabolites by unpaired t-test for this time point is provided in Figure 4G). A detailed representation of these metabolites and related pathways as a function of the entire time course is provided in the form of line plots in Figure 5.

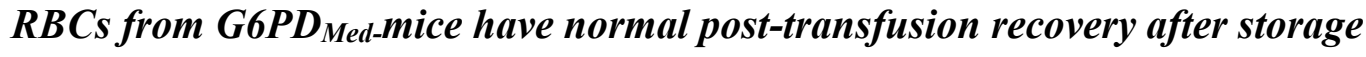


Refrigerated storage of human RBCs is an iatrogenic source of oxidant stress that is a necessary logistical component of blood banking and clinical transfusion practice. It was recently reported that when RBCs are stored from humans with G6PD deficiency and are then transfused, they have a modest (i.e., $\sim 6 \%$ ), but statistically significant, decrease in 24-hour post-transfusion recovery. To test how the hMed(-) mutation affects storage of murine RBCs, donor mice were exsanguinated and RBCs were stored using an established methodology that models the human setting(17). In this case, the 24-hour post-transfusion recovery was the same for G6PDMed- and wild-type mice (Figure 6A).

The human studies that demonstrated decreased 24-hour recoveries of transfused RBCs from G6PD-deficient donors used autologous transfusions of radiolabeled RBCs; as such, the G6PD-deficient RBCs were not only exposed to the oxidative stress of storage, but were also introduced into a recipient with potentially altered redox biology due to G6PD-deficiency. To allow for autologous transfusion studies in mice, G6PD Med- mice were crossed with B6.GFP mice. RBCs from G6PD ${ }_{\text {Med- }}$ mice were then collected, stored, and transfused into either B6.GFP mice with normal G6PD or into G6PDMed.GFP recipients; in this approach, transfused RBCs are enumerated as the GFP-negative population. No difference in 24-hour post-transfusion recovery

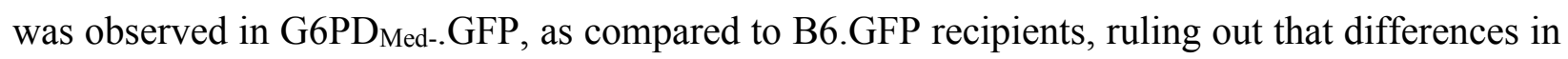
storage biology would be present if both donor and recipient were hMed(-) (Figure 6A).

Stored RBCs were also analyzed by metabolomics. At baseline (i.e., before storage), differences were observed in glycolysis and the PPP, glutathione homeostasis, and purine and amino acid metabolism (Figure 6.B); specifically, genotype-dependent alterations were detected with regards to arginine, tryptophan, and tyrosine metabolism, as gleaned by differential levels of citrulline, indoles, and dopamine, respectively (Figure 6B - right hand panel). Although increases in $\mathrm{G} 6 \mathrm{P}$ and decreases in $6 \mathrm{PG}$ were significant in $\mathrm{G6PD}_{\mathrm{Med}}$ mice both at baseline and at the end of storage (12 days), at day 12 the fold-changes for both metabolites between the two genotypes were negligible (Figure 6.C).

\section{Effects of hMed(-) on non-erythroid tissues}

G6PD deficiency has often been considered to be RBC specific, because, unlike RBCs that cannot synthesize protein, nucleated cells can compensate for decreased G6PD enzyme activity by increasing synthesis. However, in humans with G6PD deficiency, other tissues (e.g., muscle and 
endothelial cells in the pulmonary artery) have decreased G6PD activity, albeit not as severe as seen in $\operatorname{RBCs}(18,19)$. To perform a detailed analysis of multiple organs, which is not feasible in human studies, metabolomics analyses were performed on brain, heart, kidney liver, and spleen of

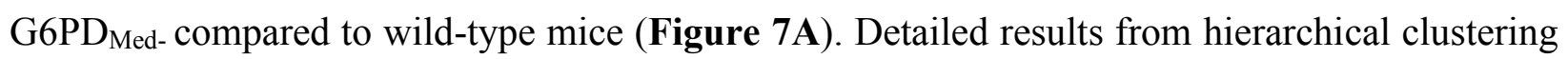
analyses of all the metabolomics data are provided for each organ in Supplementary Figures 510 and, in tabulated form, in Supplementary Table 3 (significant metabolites in Supplementary

Table 4). An overview of the multivariate analyses, including PLS-DA and heat maps reporting the top 25 significant metabolites by unpaired t-test, is provided for each organ in Figure 7-B-F.

Of note, significant decreases in carnitine-conjugated fatty acids and increases in free fatty

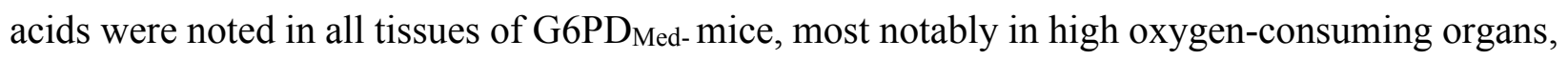
such as the brain (18.4\%), heart (11.6\%), and liver (20.4\%). There was a distinct change in betaoxidation of unsaturated fatty acids, a hallmark of oxidative metabolism and an NADPHdependent process(20), suggesting a potential mechanistic linkage between a reduced capacity to

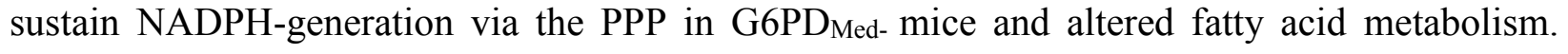
Similarly, several organs exhibited decreased levels of high-energy phosphate compounds (e.g., ADP, AMP, GDP, phosphocreatine), increased levels of amino acids (metabolized in mitochondria), and increased levels of carboxylic acids (e.g., 2-oxoglutarate and itaconate; Figure 7B-F), further suggesting depressed mitochondrial metabolism. Consistent with an apparent depression of oxidative metabolism, all organs also exhibited increased levels of lactate (Figure 7G). However, multivariate analyses (Figure 7H) indicate that - in the absence of oxidant stress the metabolic differences among organs are more pronounced than the metabolic differences observed within the same organ in G6PD $\mathrm{Med}_{\text {- }}$ and wild-type mice. 


\section{Discussion:}

Herein we describe a murine model of G6PD deficieny that overcomes the limitations of existing models, and has allowed an exploration into metabolomics of both RBCs and solid organs. These data provide novel insight into the effects of an enzymopathy that affects approximatley 5\% of all humans. The extent of the decreased activity G6PD ${ }_{\text {Med- }}$ mice, as compared to wild-type, approximates what is seen in humans (i.e. 5-10\% of normal activity) and, as is the classic finding in humans, pharmacological oxidative stress induces brisk hemolysis in vivo with hemoglobinuria. Metabolic tracing experiments demonstrated a significant decrease in glucose flux through the PPP and increased sensitivity to pharmacological oxidative stress. Although a detailed metabolomics of sold organs in humans with G6PD deficiency has not been reported, the decreased PPP in organs from the G6PD ${ }_{\text {Med- }}$ mouse is consistent with known mild decreases in G6PD organs in humans(2125).

Human G6PD-Med(-) subjects have a mildly shortened RBC circulatory lifespan (i.e., 120 days in G6PD-normal controls, as compared to 100 days in G6PD-Med(-) subjects)(26). In contrast, RBCs from the hMed(-) mice have a normal RBC circulatory lifespan. It can be speculated that the murine RBC lifespan (55 days) may not be long enough for the defects to accumulate, as they do in humans over 120 days. Conversely, in their normal state, mice have higher GSH levels than do humans, which may also mitigate the effects of G6PD defieciency in the absence of external oxidative challenge. Alternatively, other pathways involved in glutathione synthesis (e.g., ascorbate synthesis) exist in mice, but not in humans (27).

To our knowledge, this report contains the first metabolomic analysis of RBC changes after exposure to phenylhydrazine (in either WT of G6PD deficient mice); however, one caveat with this is that the changes could reflect metabolites in newly formed reticulocytes vs alterations in $\mathrm{RBCs}$ at the time of treatment. In vitro analysis of diamide treated RBCs does not suffer this issue as new RBC generation is absent. Moreover, detailed metabolomics have been reported following in vitro exposure of human G6PD-def RBCs to diamide(28). In particular, it was reported that G6PD-def RBC (but not normal RBCs) responded to diamide though depletion of GSH through oxidation (e.g decreased GSH with increasing GSSG). In response, GSH synthesis was increased as evidenced decreases in GSH precursors (e.g. $\gamma$-glutamylcysteine) and generation of byproducts of GSH sythesis (e.g. ophthalmate). ATP was also decreased in G6PD-def RBCs, which was 
interpreted as reflecting consumption of ATP by GSH synthesis. This resulted in a substantial increase in glycolytic metabolism in G6PD-def RBCs, presumably to compensate for the energy depletion of ATP consumption; however the glycolysis was abnormal in that pyruvate levels did not increase, suggestive of oxidative deactivation of pyruvate kinase. As expected, down stream metabolites of the PPP were decreased in G6PD-def RBCs. The authors of this paper also showed that the increase in glycolysis of G6PD-def RBCs in resposne to diamide correlated with increased AMPK activity that was associated with increased AMP levels due to ATP consumption.

Consistent with the above studies, when we exposed G6PDMed- mouse RBCs to diamide, we observed significant alterations of glutathione metabolism, accompanied by the inability to preserve non-deaminated high-energy purine pools in the face of decreased PPP activity. This is relevant in that RBC purine deamination by oxidant stress-activated AMP deaminase 3 is a hallmark of impaired energy metabolism, ultimately associated with a decreased ability of the RBC to circulate (29) following oxidant insult, and an overall shorter RBC lifespan in mice that are unable to replenish the ATP pool (e.g., mice with altered AMPK activity)(30). Of note, increased purine deamination by AMP deaminase 3 activation phenocopies the protective effect of G6PD deficiency on malaria infection.(31) However, a number of specific metabolic differences were

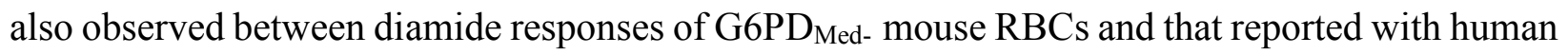
RBCs. Surprisingly, GSH levels did not decrease in mouse G6PD-def RBCs in resposne to diamide treatment; however, GSH levels did decrease in resposne to other oxidative stress (e.g. PHZ). It is worth noting that in the human studies, only a small number of humans were studied, they had a different G6PD mutation (Canton and not Mediterranean) and there may also be methodological differences. Future study will be required to assess the meaning of these respective biologies.

It was recently reported that RBCs from G6PD-deficient human volunteers stored more poorly than G6PD-normal RBCs(32). However, the decrease in post-transfusion recovery was modest $(\sim 6 \%)$. Importantly, most subjects in this study had the milder A- form of G6PD deficiency. Only a single subject was of the G6PD-Med(-) type, and unexpectedly, that subject had a post-transfusion recovery overlapping with the median measurment from the control group. The basis for this is unclear, but it indicates that the current findings with the G6PDMed-mouse are not in conflict with the one human G6PD-Med(-) who has been studied. There are conflicting 
reports regarding the extent to which G6PD activity decreases in RBC storage; it is unclear if these differences were due to differing populations or methodologies (e.g. differences in storage additives such as SAGM vs. AS-3)(33,34). However, changes in metabolism from the stored RBCs from the G6PD-deficient mice are laregly consistent with larger metabolic studies on RBCs from human donors with the G6PD-Med(-) variant (e.g. dopamine and pyruvate/lactate ratio); although post-transfusion recovereis were not reported for this group(35). Finally, recent findings from the REDS study showed similar findings during storage of units from G6PD deficient donors, although these were largely of the A-variant(36).

Although studies outside the RBC compartment in G6PD-deficient humans are limited, decreased G6PD activity in non-erythroid compartments has been reported, including in leukocytes(24), platelets(25), liver(23), and muscle(21,22). Similarly to what has been reported in humans, we show mild decreases in PPP activity in multiple organs, consistent with a mild decrease in G6PD activity. The milder deficiency in non-erythroid organs is presumably because, unlike RBCs, they have ongoing synthesis of the destabilized enzyme. More importantly, we provide a comprehensive metabolic description of mulitple organs from G6PD deficient mice, showing a role for altered PPP activation in the cross-regulation of several NADPH-dependent pathways in non-erythroid tissues. Most importantly, the current data highlight a G6PD deficiencydependent alteration in fatty acid amounts, unsaturation, and metabolism (as gleaned by the levels of free and carnitine-conjugated fatty acids). Notably, fatty acid synthesis (a key anabolic requirement for rapidly proliferating cells), desaturation, and catabolism are NADPH-dependent processes.

The role of NADPH-dependent lipid metabolism is being increasingly appreciated in tumor biology(37). Thus, our observations, combined with the appreciation of the high penetration of G6PD deficiency in human populations, raise the possibility of a previously unappreciated role for G6PD status and biology in solid tumors, analogous to what has been proposed for hematological malignancies(38). In addition, our observations suggest an indirect role for G6PD status on the capacity to metabolize fatty acids in high-oxygen demand organs (e.g., brain, heart, liver), making this model potentially useful for the study of how G6PD deficiency may affect other disorders that have been associated with G6PD deficiency in humans, including kidney disease 
and diabetes(7), pulmonary arterial hypertension(9), amytrophic lateral sclerosis(8), Huntington Disease(8), Parkinson's Disease(8), Alzheimer Disease(8), and multiple scerlosis(39).

In summary, we report a new model of G6PD deficiency in mice using a humanized enzyme of the Med- variant, and describe novel metabolic findings with regards to normal RBC biology, oxidant stress responses, and systemic alterations in peripheral organs. This new model promises to be of high utility in ongoing studies in a wide variety of biologies and pathologies in which G6PD deficiency has been implicated (e.g. aging(40), pulomnary hypertension, neurological disorders(8), exercise physiology, anti-malarial toxicology(41), mechanisms of genetic resistance to malarial infection, etc.). 


\section{Material and Methods:}

Mice: The Con-hMed(-) were generated, as described in detail in the results section, using Bruce4 ES cells. Con-hMed(-) mice were bred to mice expressing CRE under a CMV promoter (Jackson

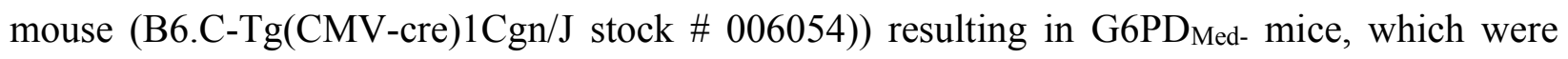
maintained through backcrossing to $\mathrm{C} 57 \mathrm{BL} / 6 \mathrm{~J}$ mice. Female heterozygous G6PD ${ }_{\text {Med- }}$ mice were bred with wild-type C57BL/6J mice such that all wild-type and G6PDMed- mice were littermate controls from the same breeding colony. Ubi-GFP (UbiC-GFP (C57BL/6-Tg(UBC-GFP)30Scha/J stock \# 004353)) and C57BL/6J mice were purchased from Jackson Labs and bred as described. All experiments were carried out under an approved IACUC protocol in the BloodworksNW vivarium and all mice were used from 2-6 months of age - for any given experiment mouse ages were matched.

RNA isolation/RT-PCR: Bone marrow was harvested from mice, and mature RBCs lysed with RBC lysis buffer. Remaining cells were resuspended in Trizol (ThermoFisher), and RNA extraction was performed per the manufacturer's instructions. RNA was converted to cDNA using the iScript gDNA clear kit (BioRad). Real-time qPCR was performed on a QuantStudio 6 Flex (Applied Biosystems), using the following Taqman primers and probes: ActB (Mm00607939_s1), Pol2Ra (Mm00839502_m1), murine G6PDx (Mm04260097_m1; spans exons 1-2), murine G6PDx (Mm00656735_g1; spans exons 12-13), human G6PD (Hs00959072_g1; spans exons 34), and human G6PD (Hs00959073; spans exons 4-5). Controls lacking reverse-transcriptase were run for every RNA preparation to control for the presence of genomic DNA; none of these samples amplified (data not shown). Data were analyzed with the QuantStudio 6 software using relative quantitation $(\Delta \Delta \mathrm{Ct})$, with $\mathrm{ActB}$ as the normalization factor and $\mathrm{B} 6$ as the control strain.

RBC lysis and hemoglobin depletion: RBC ghosts (membranes) were prepared by hypotonic lysis, followed by depletion of hemoglobin. Briefly, RBCs were washed 3 times PBS, followed by transfer of one part washed RBCs into three parts water, followed by end over end rotation for 5 min at room temperature to lyse the RBCs. Lysed RBCs were then mixed 1:1 with Hemoglobind (Biotech Support Group), followed by end over end rotation for $10 \mathrm{~min}$ at room temperature. 
Hemoglobind and bound hemoglobin were pelleted by centrifugation, and supernatants subjected to an additional hemoglobin depletion with hemoglobind. Supernatants were used for western blotting (below).

Western blotting: Hemoglobin-depleted supernatants were electrophoresed on 4-12\% NuPAGE Bis-Tris gels under reducing conditions, and transferred to PVDF membranes. As a control, recombinant human G6PD protein (Abcam, cat\#NP0007) was also included. Membranes were blocked in $2.5 \%$ milk/2.5\% BSA in TBST, and probed with rabbit anti-human/mouse G6PD (Abcam, cat\# EPR20688) or rabbit anti-Actin (Cell Signaling Technology, cat\#4970), and imaged by standard chemiluminescence techniques on an ImageQuat-800 (GE).

G6PD Activity Assay: For the quantitative determination of G6PD activity, the Glucose-6Phosphate Dehydrogenase Reagent Set was used (Pointe Scientific, cat\# G7583180). Following the manufacturer's protocol, 10ul of whole non-washed RBCs were assayed using a heated cuvette spectrophotometer (Nanophotometer C40, Implen); $\mathrm{Hb}(\mathrm{g} / \mathrm{dL}$ ) was measured using 70ul whole, unwashed RBCs on an ABLX90 (Radiometer). Results are presented as G6PD activity (U/g Hb).

Statistics for RT-PCR: Analysis using two-way ANOVA with Sidak post-hoc testing revealed no significant differences in mRNA levels between WT and G6PD ${ }^{\mathrm{Med}-}$ mice for either Pol2RA or Mm04260097_m1.

Blood Storage and Transfusion Studies: Blood storage, transfusion, and determination of posttransfusion recovery were carried out as described in detail in previous studies $(17,42)$.

RBC lifespan determination and oxidant stress challenge: RBC lifespan determination was carried out by the biotinylation method(14), and as described in detail in previous studies(43). Phenylhydrazine was given to mice through 3 intraperitoneal injections of $0.01 \mathrm{mg} / \mathrm{g}$ administered 12 hours apart. 


\section{Metabolomics}

Sample processing and metabolite extraction: Fifty $\mu \mathrm{L}$ of frozen RBC aliquots or $10 \mathrm{mg}$ of snap frozen organ tissues were extracted in $450 \mathrm{ul}$ or $1 \mathrm{ml}$, respectively, of ice cold methanol:acetonitrile:water $(5: 3: 2 \mathrm{v} / \mathrm{v})$. Samples were agitated at $4^{\circ} \mathrm{C}$ for $30 \mathrm{~min}$ followed by centrifugation at $10,000 \times \mathrm{g}$ for $10 \mathrm{~min}$ at $4^{\circ} \mathrm{C}$, as described (44). Protein pellets were discarded, and supernatants were stored at $-80^{\circ} \mathrm{C}$ prior to metabolomic analysis.

Ultra-High-Pressure Liquid Chromatography-Mass Spectrometry (MS) metabolomics: Samples were randomized and $10 \mu \mathrm{l}$ aliquots of extracts were injected using a UHPLC system (Vanquish, Thermo, San Jose, CA, USA) and run on a Kinetex C18 column (150 x $2.1 \mathrm{~mm}, 1.7 \mu \mathrm{m}-$ Phenomenex, Torrance, CA, USA) at $250 \mu \mathrm{l} / \mathrm{min}$ (isocratic: 5\% Optima acetonitrile, 95\% Optima $\mathrm{H} 2 \mathrm{O}, 0.1 \%$ formic acid)(45) and $400 \mu \mathrm{l} / \mathrm{min}$ (5 or $17 \mathrm{~min}$ gradient $5-95 \% \mathrm{~B}$; A:water $+0.1 \%$ formic acid, B: acetonitrile $+0.1 \%$ formic acid)(46, 47). Formic acid in mobile phases was replaced by 1 $\mathrm{mM}$ ammonium acetate for negative mode runs. The UHPLC system was coupled online with a $\mathrm{Q}$ Exactive mass spectrometer (Thermo, Bremen, Germany), scanning in Full MS mode ( $2 \mu$ scans) at 70,000 resolution in the $60-900 \mathrm{~m} / \mathrm{z}$ range operated in either polarity mode. Eluates were subjected to electrospray ionization (ESI) in positive and negative ion modes (separate runs) with $4 \mathrm{kV}$ spray voltage, 15 sheath gas, and 5 auxiliary gas. Separate Top15 ddMS2 runs were performed on tech mixes composed of $10 \mu \mathrm{l}$ of each extract from each one of the samples in the batch. Chromatographic and MS technical stability were assessed by determining CVs $<10 \%$ for metabolites in mixed controls run every 5 injections in the queue. MS1 and data-dependent MS2 acquisition(48), data analysis, and elaboration were performed, as described(46, 47). Graphs and statistical analyses (either t-test or repeated measures or two-way ANOVA) were generated with GraphPad Prism 8.1.2 (GraphPad Software, Inc, La Jolla, CA), GENE E (Broad Institute, Cambridge, MA, USA), and MetaboAnalyst 4.0(49). 
Author Contributions: ADA, SLS, EAH, ROF, MK, TT and JCZ concieved of the studiess, designed experiments and interpreted data. $\mathrm{HLH}, \mathrm{AMH}, \mathrm{BB}, \mathrm{MJW}$, and XF carried out experiments, generated data and interperted data. All authors were actively involved in the writing of the manuscript and the interpretation of data.

Acknowledgements: This research was supported by funds from the RM1GM131968 (ADA) from the National Institute of General and Medical Sciences, and R01HL146442 (ADA), R01HL149714 (ADA), R01HL148151 (SLS, ADA, JCZ), R21HL150032 (ADA), from the National Heart, Lung, and Blood Institute.

Disclosure of Conflicts of Interest: Although unrelated to the studies in the current manuscript, $\mathrm{AD}$ is the founder of Omix Technologies and Altis Biosciences LLD. AD and SLS are consultants for Hemanext Inc. BloodworksNW, where the G6PD mouse was generated, has filed intellectual property on use of this animal as a tool for screening toxicology of novel drugs. The authors have no other conflicts of interest to declare. 


\section{References:}

1. Luzzatto L, Nannelli C, and Notaro R. Glucose-6-Phosphate Dehydrogenase Deficiency. Hematol Oncol Clin North Am. 2016;30(2):373-93.

2. Beutler E, and Vulliamy TJ. Hematologically important mutations: glucose-6-phosphate dehydrogenase. Blood Cells Mol Dis. 2002;28(2):93-103.

3. Minucci A, Moradkhani K, Hwang MJ, Zuppi C, Giardina B, and Capoluongo E. Glucose-6phosphate dehydrogenase (G6PD) mutations database: review of the "old" and update of the new mutations. Blood Cells Mol Dis. 2012;48(3):154-65.

4. Luzzatto L, and Arese P. Favism and Glucose-6-Phosphate Dehydrogenase Deficiency. N Engl J Med. 2018;378(1):60-71.

5. Belfield KD, and Tichy EM. Review and drug therapy implications of glucose-6-phosphate dehydrogenase deficiency. Am J Health Syst Pharm. 2018;75(3):97-104.

6. Thomas JE, Kang S, Wyatt CJ, Kim FS, Mangelsdorff AD, and Weigel FK. Glucose-6-Phosphate Dehydrogenase Deficiency is Associated with Cardiovascular Disease in U.S. Military Centers. Tex Heart Inst J. 2018;45(3):144-50.

7. Spencer NY, and Stanton RC. Glucose 6-phosphate dehydrogenase and the kidney. Curr Opin Nephrol Hypertens. 2017;26(1):43-9.

8. Tiwari M. Glucose 6 phosphatase dehydrogenase (G6PD) and neurodegenerative disorders: Mapping diagnostic and therapeutic opportunities. Genes Dis. 2017;4(4):196-203.

9. Kurdyukov S, Eccles CA, Desai AA, Gonzalez-Garay M, Yuan JX, Garcia JGN, et al. New cases of Glucose-6-Phosphate Dehydrogenase deficiency in Pulmonary Arterial Hypertension. PLoS One. 2018;13(8):e0203493.

10. Baader SL, Schilling ML, Rosengarten B, Pretsch W, Teutsch HF, Oberdick J, et al. Purkinje cell lineage and the topographic organization of the cerebellar cortex: a view from $\mathrm{X}$ inactivation mosaics. Developmental biology. 1996;174(2):393-406.

11. Pretsch W, Charles DJ, and Merkle S. X-linked glucose-6-phosphate dehydrogenase deficiency in Mus musculus. Biochemical genetics. 1988;26(1-2):89-103.

12. Au SW, Gover S, Lam VM, and Adams MJ. Human glucose-6-phosphate dehydrogenase: the crystal structure reveals a structural NADP(+) molecule and provides insights into enzyme deficiency. Structure. 2000;8(3):293-303.

13. Boonyuen U, Chamchoy K, Swangsri T, Junkree T, Day NPJ, White NJ, et al. A trade off between catalytic activity and protein stability determines the clinical manifestations of glucose-6phosphate dehydrogenase (G6PD) deficiency. Int J Biol Macromol. 2017;104(Pt A):145-56.

14. Hoffmann-Fezer G, Maschke H, Zeitler HJ, Gais P, Heger W, Ellwart J, et al. Direct in vivo biotinylation of erythrocytes as an assay for red cell survival studies. Ann Hematol. 1991;63(4):214-7.

15. Mohler DN, and Williams WJ. The effect of phenylhydrazine on the adenosine triphosphate content of normal and glucose-6-phosphate dehydrogenase-deficient human blood. J Clin Invest. 1961;40:1735-42.

16. Zhang Y, Lee JH, Paull TT, Gehrke S, D'Alessandro A, Dou Q, et al. Mitochondrial redox sensing by the kinase ATM maintains cellular antioxidant capacity. Sci Signal. 2018;11(538).

17. Gilson CR, Kraus TS, Hod EA, Hendrickson JE, Spitalnik SL, Hillyer CD, et al. A novel mouse model of red blood cell storage and posttransfusion in vivo survival. Transfusion. 2009;49(8):1546-53. 
18. Stanton RC. Glucose-6-phosphate dehydrogenase, NADPH, and cell survival. IUBMB Life. 2012;64(5):362-9.

19. Serpillon S, Floyd BC, Gupte RS, George S, Kozicky M, Neito V, et al. Superoxide production by $\mathrm{NAD}(\mathrm{P}) \mathrm{H}$ oxidase and mitochondria is increased in genetically obese and hyperglycemic rat heart and aorta before the development of cardiac dysfunction. The role of glucose-6-phosphate dehydrogenase-derived NADPH. Am J Physiol Heart Circ Physiol. 2009;297(1):H153-62.

20. Smeland TE, Nada M, Cuebas D, and Schulz H. NADPH-dependent beta-oxidation of unsaturated fatty acids with double bonds extending from odd-numbered carbon atoms. Proc Natl Acad Sci U S A. 1992;89(15):6673-7.

21. Bresolin N, Bet L, Moggio M, Meola G, Comi G, Gilardi A, et al. Muscle G6PD deficiency. Lancet. 1987;2(8552):212-3.

22. Bresolin N, Bet L, Moggio M, Meola G, Fortunato F, Comi G, et al. Muscle glucose-6-phosphate dehydrogenase deficiency. J Neurol. 1989;236(4):193-8.

23. Marks PA, Gross RT, and Hurwitz RE. Gene action in erythrocyte deficiency of glucose-6phosphate dehydrogenase: tissue enzyme-levels. Nature. 1959;183(4670):1266-7.

24. Ramot B, Fisher S, Szeinberg A, Adam A, Sheba C, and Gafni D. A study of subjects with erythrocyte glucose-6-phosphate dehydrogenase deficiency. II. Investigation of leukocyte enzymes. J Clin Invest. 1959;38:2234-7.

25. Ramot B, Szeinberg A, Adam A, Sheba C, and Gafni D. A study of subjects with erythrocyte glucose-6-phosphate dehydrogenase deficiency: investigation of platelet enzymes. $J$ Clin Invest. 1959;38:1659-61.

26. Bernini L, Latte B, Siniscalco M, Piomelli S, Spada U, Adinolfi M, et al. Survival of $51 \mathrm{Cr}$-Labelled Red Cells in Subjects with Thalassaemia-Trait or G6pd Deficiency or Both Abnormalities. Br J Haematol. 1964;10:171-80.

27. Pallotta V, Gevi F, D'Alessandro A, and Zolla L. Storing red blood cells with vitamin C and Nacetylcysteine prevents oxidative stress-related lesions: a metabolomics overview. Blood Transfus. 2014;12(3):376-87.

28. Tang HY, Ho HY, Wu PR, Chen SH, Kuypers FA, Cheng ML, et al. Inability to maintain GSH pool in G6PD-deficient red cells causes futile AMPK activation and irreversible metabolic disturbance. Antioxid Redox Signal. 2015;22(9):744-59.

29. Nemkov T, Sun K, Reisz JA, Song A, Yoshida T, Dunham A, et al. Hypoxia modulates the purine salvage pathway and decreases red blood cell and supernatant levels of hypoxanthine during refrigerated storage. Haematologica. 2018;103(2):361-72.

30. Wang S, Dale GL, Song P, Viollet B, and Zou MH. AMPKalpha1 deletion shortens erythrocyte life span in mice: role of oxidative stress. J Biol Chem. 2010;285(26):19976-85.

31. Hortle E, Nijagal B, Bauer DC, Jensen LM, Ahn SB, Cockburn IA, et al. Adenosine monophosphate deaminase 3 activation shortens erythrocyte half-life and provides malaria resistance in mice. Blood. 2016;128(9):1290-301.

32. Francis RO, D'Alessandro A, Eisenberger A, Soffing M, Yeh R, Coronel E, et al. Donor glucose-6phosphate dehydrogenase deficiency decreases blood quality for transfusion. $J$ Clin Invest. 2020;130(5):2270-85.

33. Francis RO, Jhang J, Hendrickson JE, Zimring JC, Hod EA, and Spitalnik SL. Frequency of glucose6-phosphate dehydrogenase-deficient red blood cell units in a metropolitan transfusion service. Transfusion. 2013;53(3):606-11.

34. Messana I, Ferroni L, Misiti F, Girelli G, Pupella S, Castagnola M, et al. Blood bank conditions and RBCs: the progressive loss of metabolic modulation. Transfusion. 2000;40(3):353-60. 
35. Tzounakas VL, Kriebardis AG, Georgatzakou HT, Foudoulaki-Paparizos LE, Dzieciatkowska M, Wither MJ, et al. Glucose 6-phosphate dehydrogenase deficient subjects may be better "storers" than donors of red blood cells. Free Radic Biol Med. 2016;96:152-65.

36. D'Alessandro A, Fu X, Kanias T, Reisz JA, Culp-Hill R, Guo Y, et al. Donor sex, age and ethnicity impact stored red blood cell antioxidant metabolism through mechanisms in part explained by glucose 6-phosphate dehydrogenase levels and activity. Haematologica. 2020.

37. Carracedo A, Cantley LC, and Pandolfi PP. Cancer metabolism: fatty acid oxidation in the limelight. Nat Rev Cancer. 2013;13(4):227-32.

38. Gregory MA, D'Alessandro A, Alvarez-Calderon F, Kim J, Nemkov T, Adane B, et al. ATM/G6PDdriven redox metabolism promotes FLT3 inhibitor resistance in acute myeloid leukemia. Proc Natl Acad Sci U S A. 2016;113(43):E6669-E78.

39. Meloni T, Forteleoni G, Aiello I, Pirastru MI, Sanna G, Meloni GF, et al. Glucose-6-phosphate dehydrogenase deficiency and multiple sclerosis in northern Sardinia. Neuroepidemiology. 1993;12(6):350-2.

40. Nobrega-Pereira S, Fernandez-Marcos PJ, Brioche T, Gomez-Cabrera MC, Salvador-Pascual A, Flores JM, et al. G6PD protects from oxidative damage and improves healthspan in mice. Nat Commun. 2016;7:10894.

41. Baird JK. 8-Aminoquinoline Therapy for Latent Malaria. Clin Microbiol Rev. 2019;32(4).

42. de Wolski K, Fu X, Dumont L, Roback JD, Waterman H, Odem-Davis K, et al. Metabolic pathways that correlate with post-transfusion circulation of stored murine red blood cells. Haematologica. 2016;101(5):578-86.

43. Zimring JC, Smith N, Stowell SR, Johnsen JM, Bell LN, Francis RO, et al. Strain-specific red blood cell storage, metabolism, and eicosanoid generation in a mouse model. Transfusion. 2014;54(1):137-48.

44. Reisz JA, Wither MJ, Dzieciatkowska M, Nemkov T, Issaian A, Yoshida T, et al. Oxidative modifications of glyceraldehyde 3-phosphate dehydrogenase regulate metabolic reprogramming of stored red blood cells. Blood. 2016;128(12):e32-42.

45. Nemkov T, Hansen KC, and D'Alessandro A. A three-minute method for high-throughput quantitative metabolomics and quantitative tracing experiments of central carbon and nitrogen pathways. Rapid Commun Mass Spectrom. 2017;31(8):663-73.

46. Nemkov T, Reisz JA, Gehrke S, Hansen KC, and D'Alessandro A. High-Throughput Metabolomics: Isocratic and Gradient Mass Spectrometry-Based Methods. Methods Mol Biol. 2019;1978:13-26.

47. Reisz JA, Zheng C, D'Alessandro A, and Nemkov T. Untargeted and Semi-targeted Lipid Analysis of Biological Samples Using Mass Spectrometry-Based Metabolomics. Methods Mol Biol. 2019;1978:121-35.

48. Gehrke S, Rice S, Stefanoni D, Wilkerson RB, Nemkov T, Reisz JA, et al. Red Blood Cell Metabolic Responses to Torpor and Arousal in the Hibernator Arctic Ground Squirrel. J Proteome Res. 2019;18(4):1827-41.

49. Chong J, Soufan O, Li C, Caraus I, Li S, Bourque G, et al. MetaboAnalyst 4.0: towards more transparent and integrative metabolomics analysis. Nucleic Acids Res. 2018;46(W1):W486-W94. 


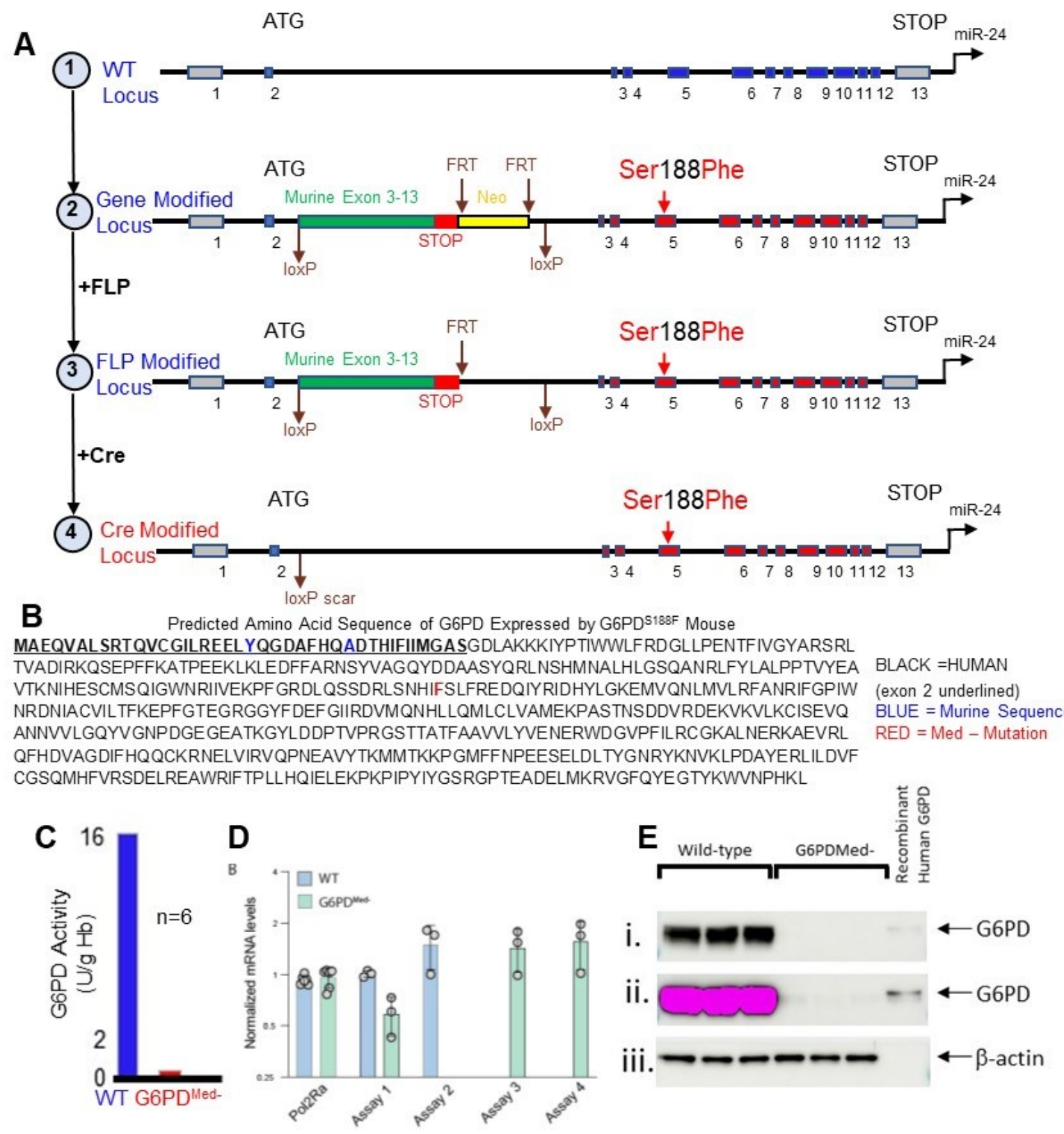

Figure 1 - Generation of a new G6PD deficient mouse model. (A) Schematic representation of the WT G6PD locus (top) and related modifications (bottom). (B) Predicted protein sequence of knocked in G6PD gene. (C) G6PD activity in wild-type vs knockin mouse. (D) mouse vs. human mRNA levles in G6PD Med- and WT mice, (E) Western blot analysis of cytoplasm from RBCs. Because the G6PD gene is X lined, males were used in all cases. G6PD Med- mice were hemizygous for the knocked in human gene, WT mice are litter mate contols with the mouse G6PD. 


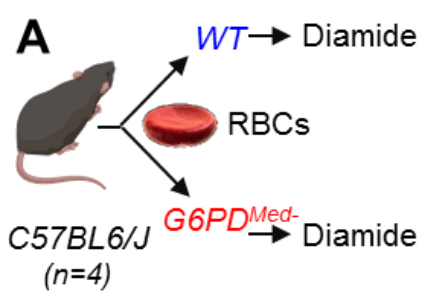

E
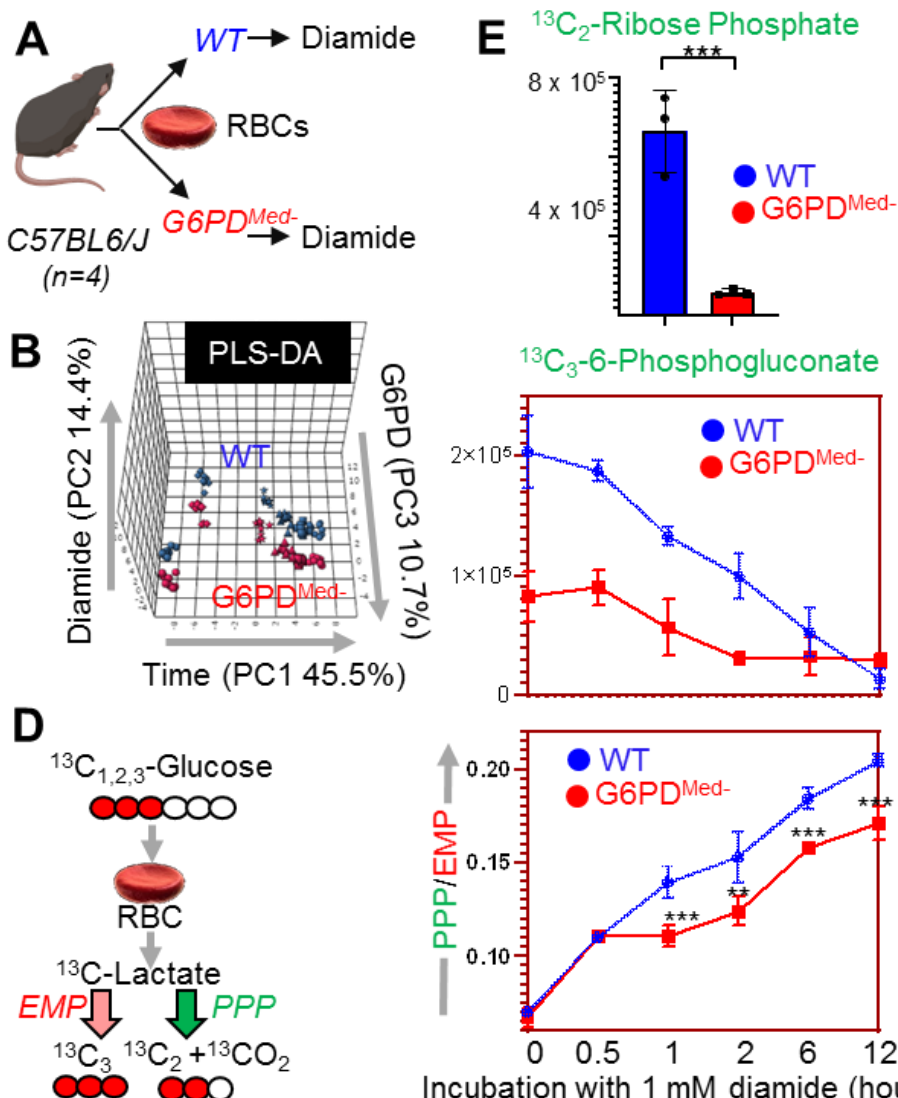
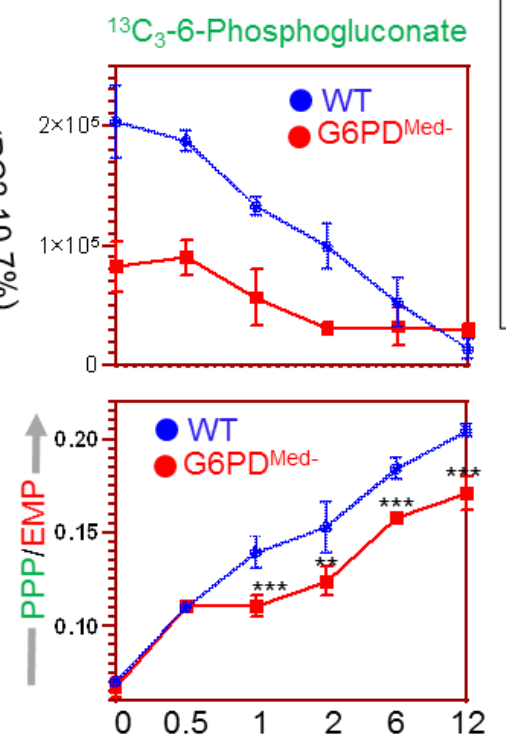

Incubation with $1 \mathrm{mM}$ diamide (hours)

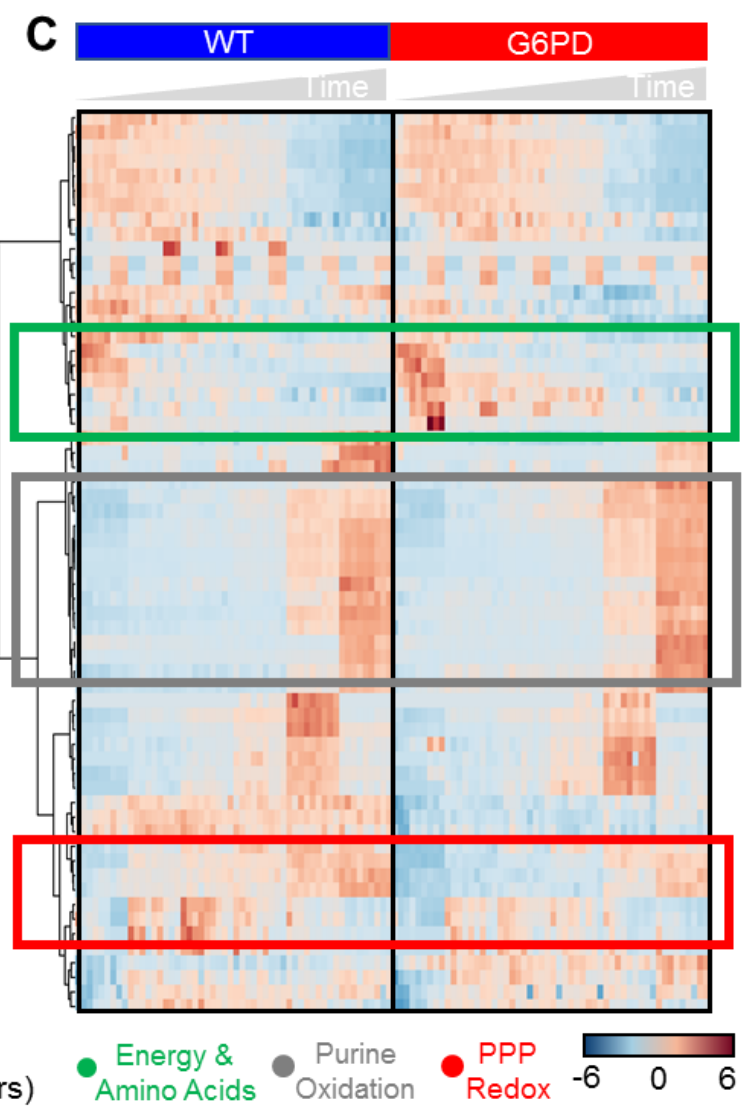

Figure 2 - Metabolic effect of diamide challenge in RBCs from WT and G6PD deficient mice. RBCs from WT and G6PD def mice were incubated with $1 \mathrm{mM}$ diamide (A). RBCs were tested at $0 \mathrm{~min}$ (no diamide), 30min, $1 \mathrm{~h}, 2 \mathrm{~h}, 6 \mathrm{~h}$ and $12 \mathrm{~h}$ from incubation with diamide prior to metabolomics analysis. Multivariate analyses included PLS-DA (B) and hierarchical clustering analysis (C) clearly indicate a time-dependent effect of the treatment on RBCs (PC1 explaining 45.5\% of the total variance) and highlighted the impact of G6PD activity (PC3 - 10.7\% of the total variance). Significant metabolites by repeated measures one-way ANOVA are shown in the heat map in $\mathbf{C}$. In $\mathbf{D}$, the experiment was repeated by incubating RBCs in presence of $1,2,3-{ }^{13} \mathrm{C}_{3}$-glucose. By quantifying isotopologues $\mathrm{M}+2$ and $\mathrm{M}+3$ of lactate (and the relative ratio), fluxes through Pentose Phosphate Pathway vs Glycolysis can be determined, confirmed a significantly lower activation of this pathway in RBCs from G6PD def mice upon diamide challenge. 


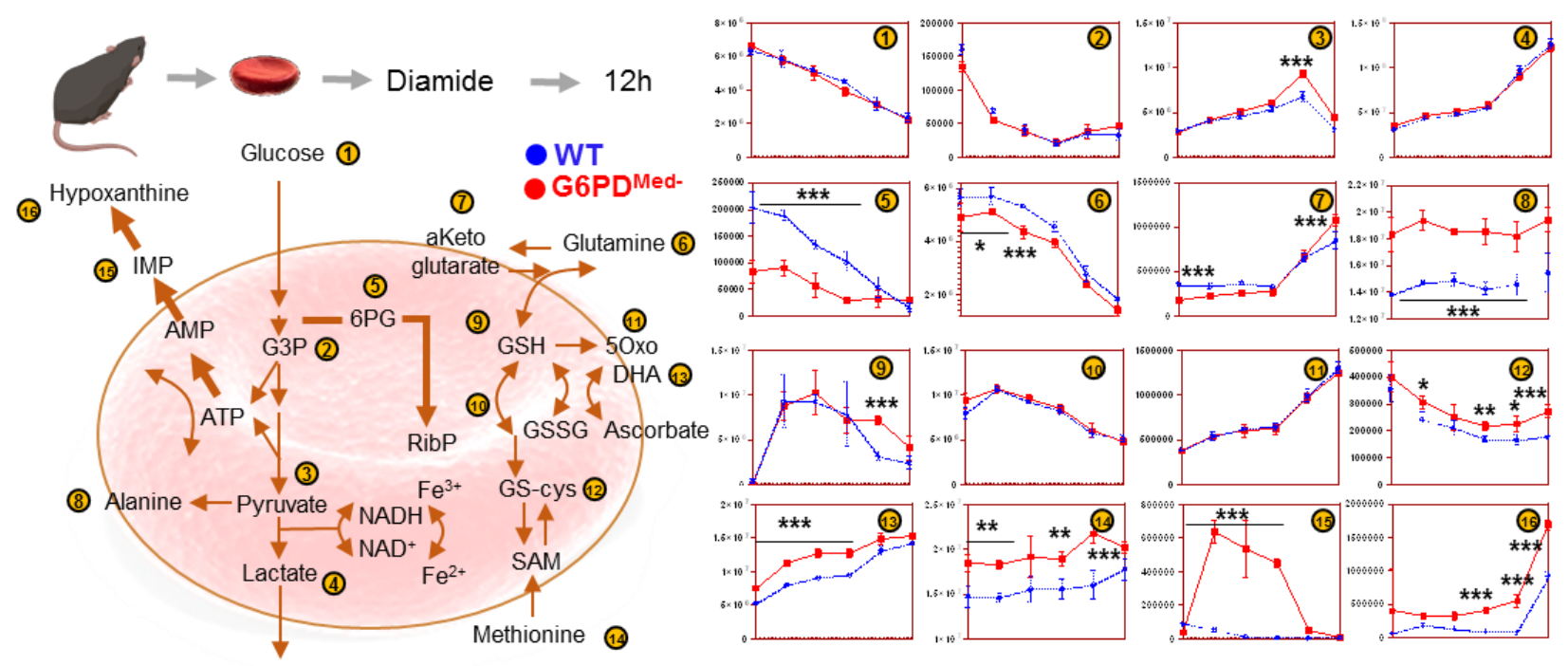

Figure 3 - Overview of glycolysis, pentose phosphate pathway, glutathione metabolism and recycling, purine oxidation in RBCs from WT (blue) and G6PD def (red) mouse red blood cells $(n=3)$ treated with $1 \mathrm{mM}$ diamide for up to 12 hours. Time points tested were 0 (no diamide), 30min, $1 \mathrm{~h}, 2 \mathrm{~h}, 6 \mathrm{~h}$ and $12 \mathrm{~h}$ from incubation with diamide. Significantly increased pyruvate/lactate ratios and alanine accumulation in G6PD def mouse RBCs is consistent with an increased utilization of NADH by other enzymes than lactate dehydrogenase, consistent with higher levels/activity of methemoglobin reductase in prior human studies on Med- G6PD def RBCs (Tzounakas et al. 2016 - https://www.ncbi.nlm.nih.gov/pubmed/27094493). 

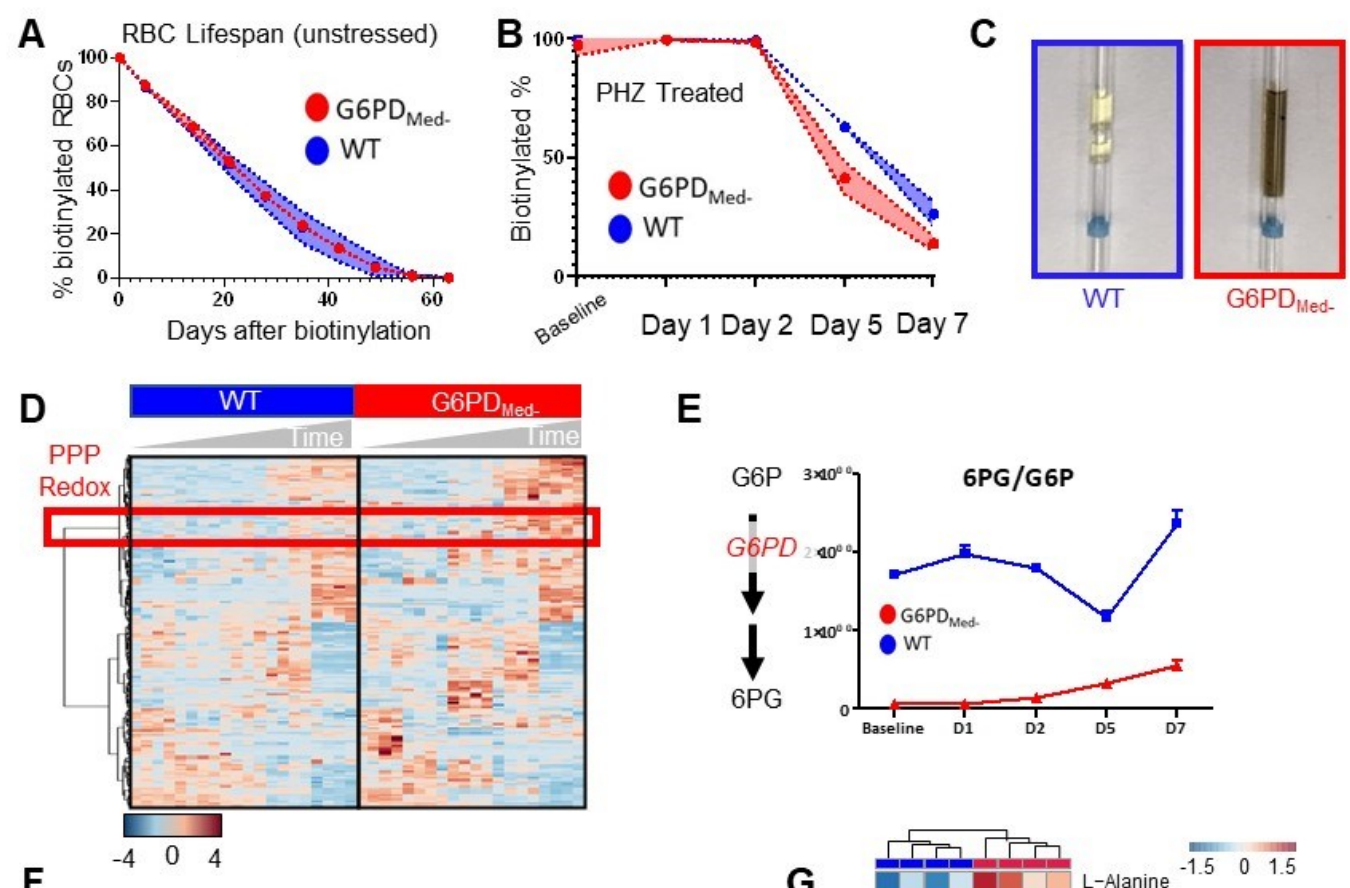

$\mathbf{F}$
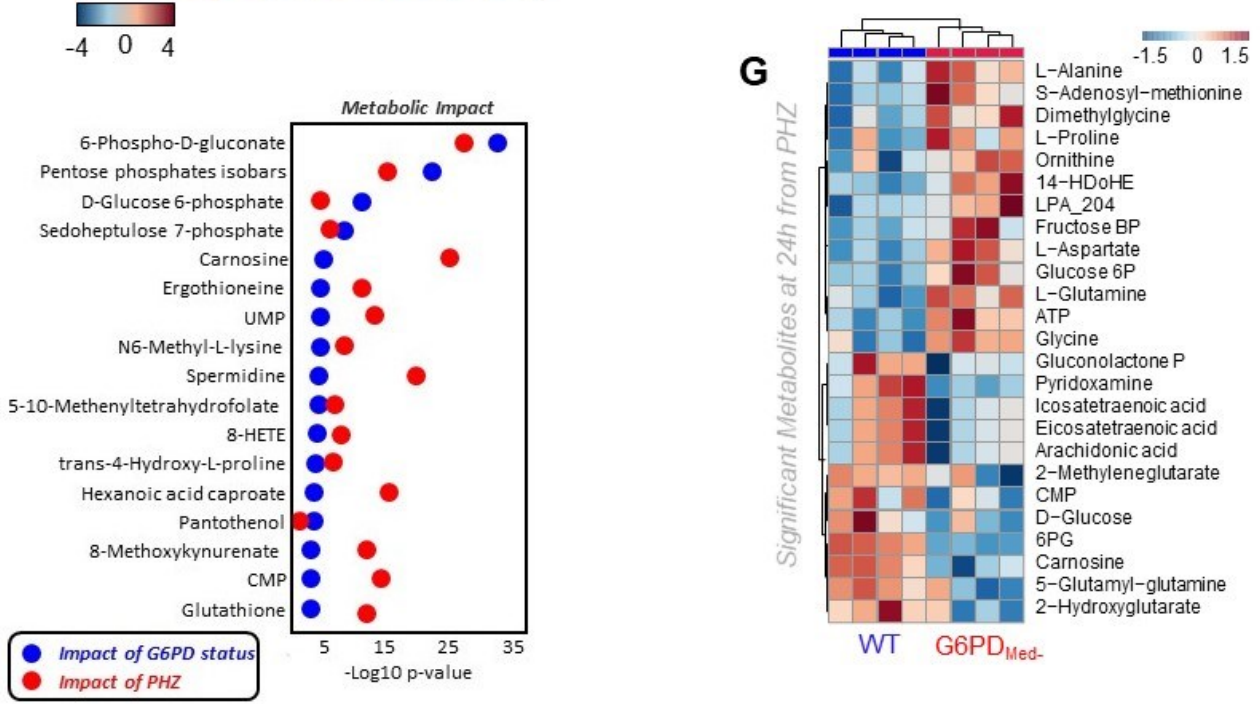

Figure 4 - Phenylhydrazine (PHZ) induces brisk hemoglobinuria in G6PD def mouse RBCs, but not in WT (A). Biotinylation experimetns showed significant decreases in circulating RBC survival after the $2^{\text {nd }} \mathrm{PHZ}$ treatment in G6PD deficient mice (B). G6PD def RBCs at baseline and upon treatment with PHZ for up to 7 days are incapable of activating the PPP (C), as determined by the ratios of G6PD product/substrate, a proxy for the determination of the enzyme activity by law of mass action, as described (Zhang et al. Sci Signaling 2018 - https:/www.ncbi.nlm.nih.gov/pubmed/29991649) (D). Significant metabolic changes were noted between the two groups upon prolonged treatment with PHZ (C). Pathway analysis highlighted 4 PPP-related metabolites on the top 5 of significant metabolic changes by two way ANOVA (first column: impact of G6PD deficiency; second column: impact of PHZ treatment; third column: interactions: (E-F)). These changes mostly involved oxidant stress and sulfur metabolism-related pathways (ergothioneine, glutathione, oxylipins) and ultimately resulted in distinct metabolic phenotypes as a function of PHZ treatment and between WT and G6PD def RBCs. These differences are clear when comparing the time point at 24h from PHZ injection in WT and G6PD def mice as highlighted in the heat map in $\mathrm{F}$. 


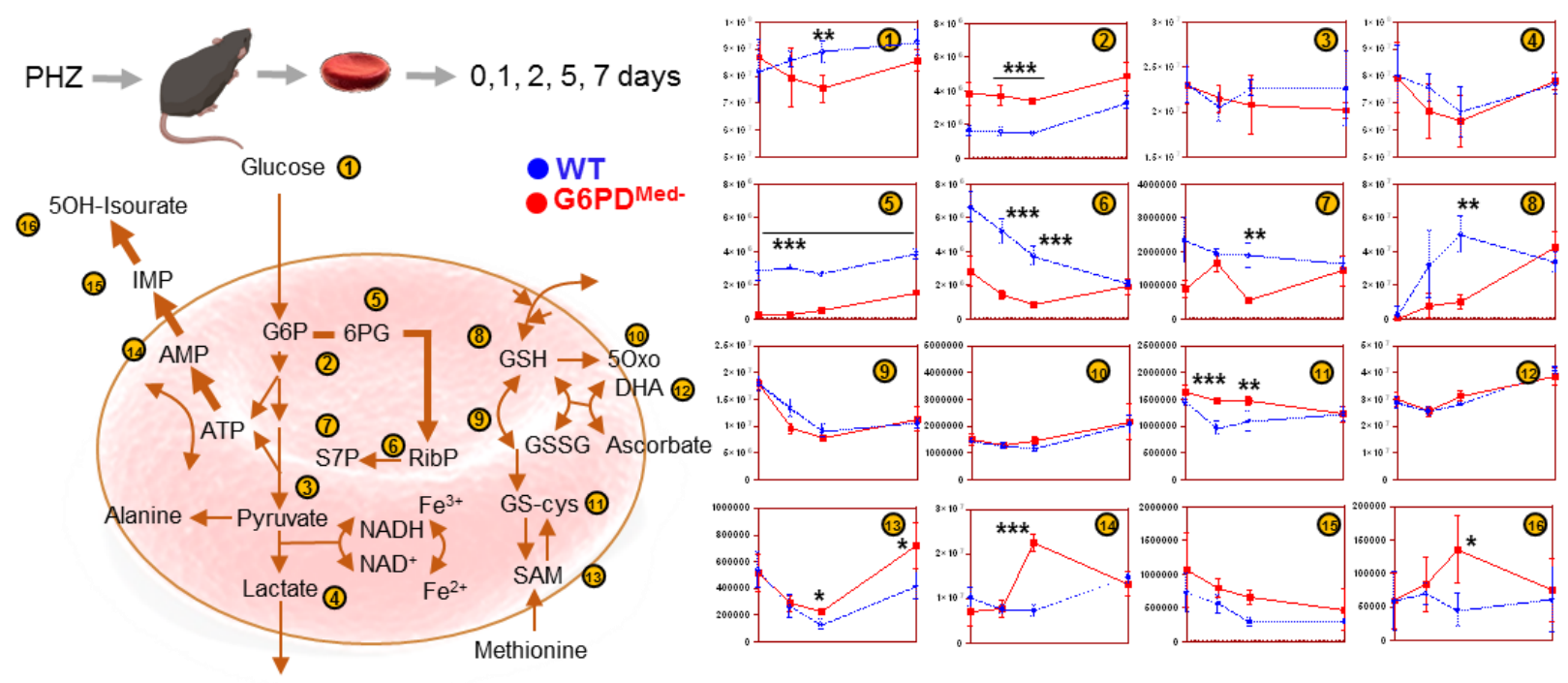

Figure 5 - Metabolic impact of PHZ treatment in vivo on WT and G6PD def mouse RBCs. Overview of glycolysis, pentose phosphate pathway, glutathione metabolism and recycling, purine oxidation in RBCs from WT (blue) and G6PD def (red) mouse red blood cells ( $\mathrm{n}=3$ ) treated with $1 \mathrm{mM}$ phenylhydrazine (PHZ) for up to 7 days. Time points tested were 0 (no PHZ), 1, 2, 5 and 7 days from treatment with PHZ. 


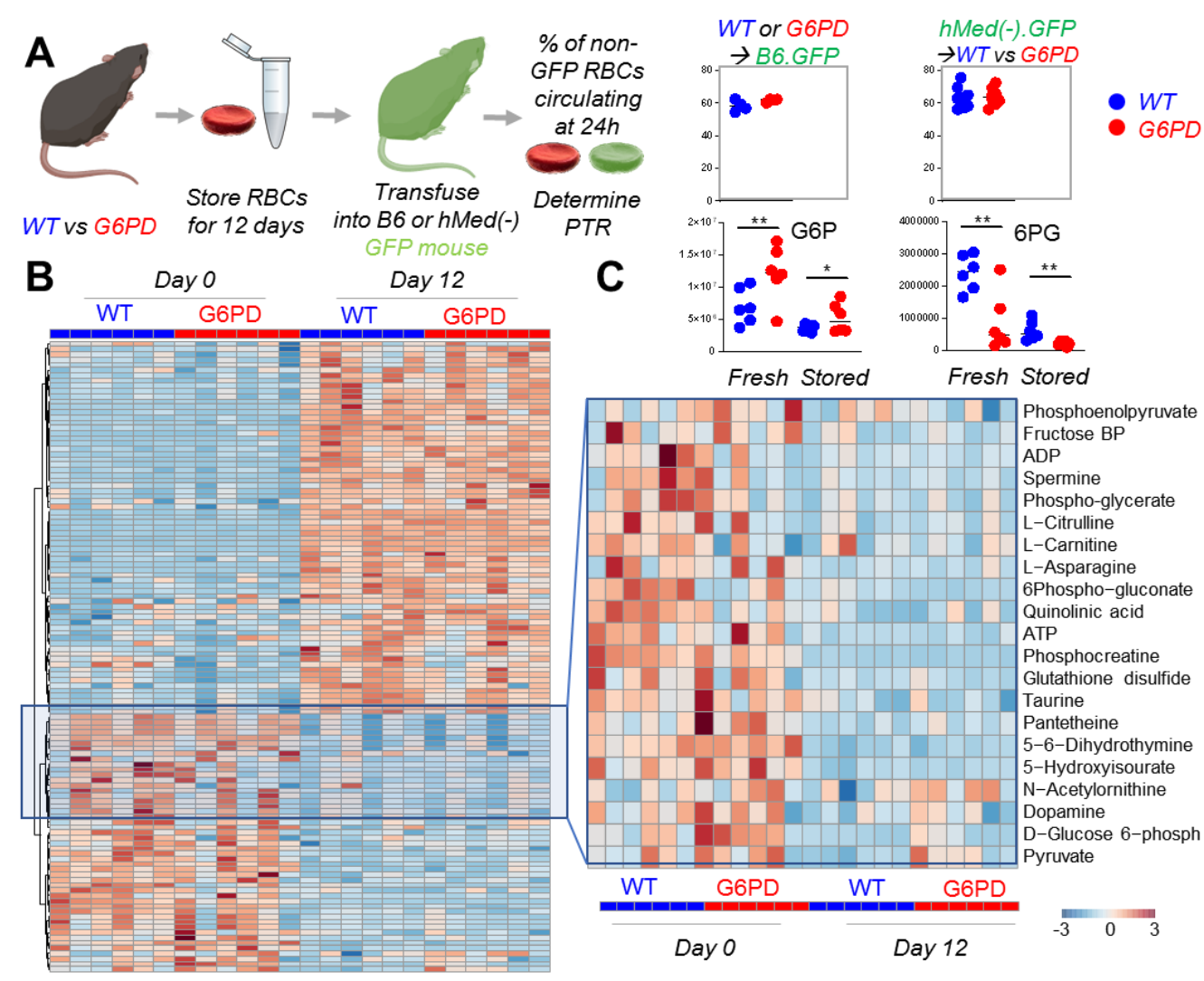

Figure 6 - G6PD deficient mouse RBCs have comparable metabolism and post-transfusion recovery to wild-type RBCs at the end of storage. RBCs from WT and hMed(-) G6PD mice $(n=6)$ were stored under conditions mimicking storage in the blood bank for 12 days $(\mathbf{A})$. At the end of storage, RBCs were transfused into Ubi-GFP mice and flow cytometry studies were performed to determine the percentage of transfused RBCs circulating at $24 \mathrm{~h}$ from transfusion, which was determined to be comparable between the two groups. Transfusing hMed(-).GFP into WT or hMed(-) non-GFP recipient did not impact the PTR \% measurement. Metabolic phenotypes of G6PD deficient RBCs showed some significant differences at baseline (especially with respect to glycolysis, the pentose phosphate pathway and glutathione homeostasis (B). However, these changes were not appreciable by the end of storage. A detail for glucose 6phosphate (G6P) and 6-phosphogluconate (6PG) is provided in (C) 

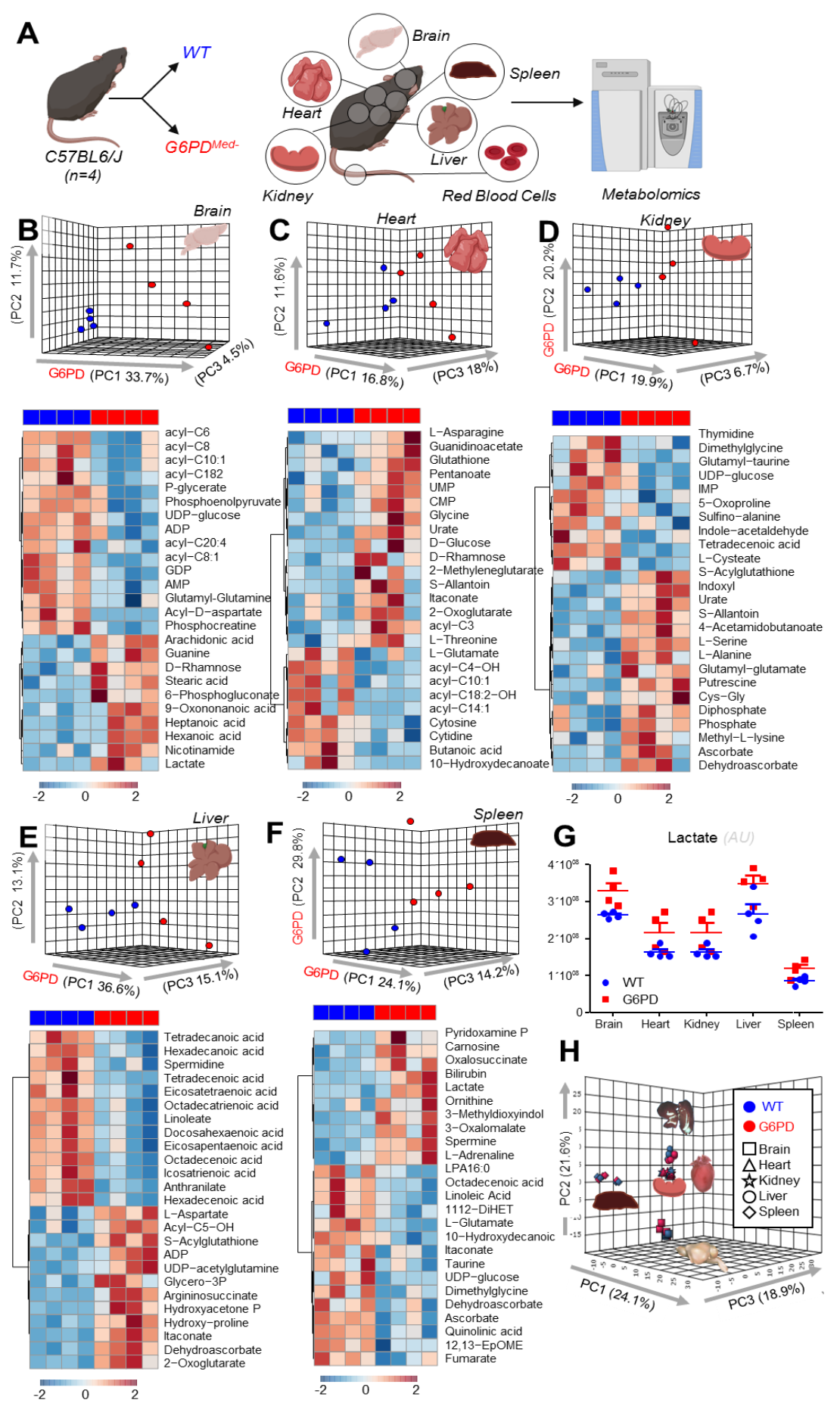

Figure 7 - Metabolic analyses of brain, liver, heart, kidney and spleen from WT and G6PD def mice (A). Significant metabolic changes were observed in all the organ tested from transgenic mice, compared to WT controls (B-F), as highlighted by multivariate principal component and hierarchical clustering analyses (only the top 25 significant metabolites - T-test are shown). The majority of metabolic changes in organs of G6PD subjects related to fatty acid metabolism and acyl-carnitines, followed by amino acid metabolism and glycolysis. Lactate levels were significantly lower in all organs of G6PD deficient mice $(\mathbf{G})$, though organ-to-organ metabolic differences overweighed differences between WT and transgenic mice $(\mathbf{H})$. 